\title{
Optimal left ventricular lead position assessed with phase analysis on gated myocardial perfusion SPECT
}

\author{
Mark J. Boogers • Ji Chen • Rutger J. van Bommel • C. Jan Willem Borleffs • \\ Petra Dibbets-Schneider • Bernies van der Hiel • Imad Al Younis • Martin J. Schalij • \\ Ernst E. van der Wall • Ernest V. Garcia • Jeroen J. Bax
}

Received: 6 June 2010 /Accepted: 6 September 2010 /Published online: 17 October 2010

(C) The Author(s) 2010. This article is published with open access at Springerlink.com

\begin{abstract}
Purpose The aim of the current study was to evaluate the relationship between the site of latest mechanical activation as assessed with gated myocardial perfusion SPECT (GMPS), left ventricular (LV) lead position and response to cardiac resynchronization therapy (CRT).

Methods The patient population consisted of consecutive patients with advanced heart failure in whom CRT was currently indicated. Before implantation, 2-D echocardiography and GMPS were performed. The echocardiography was performed to assess LV end-systolic volume (LVESV), LV end-diastolic volume (LVEDV) and LV ejection fraction (LVEF). The site of latest mechanical activation was assessed by phase analysis of GMPS studies and related to LV lead position on fluoroscopy. Echocardiography was
\end{abstract}

M. J. Boogers · R. J. van Bommel • C. J. W. Borleffs •

M. J. Schalij • E. E. van der Wall · J. J. Bax $(\bowtie)$

Department of Cardiology, Leiden University Medical Center,

Albinusdreef 2,

2333 ZA Leiden, The Netherlands

e-mail: j.j.bax@lumc.nl

M. J. Boogers

e-mail: j.m.j.boogers@lumc.nl

M. J. Boogers

The Interuniversity Cardiology Institute of the Netherlands,

Utrecht, The Netherlands

J. Chen · E. V. Garcia

Department of Radiology, Emory University School of Medicine,

Atlanta, GA, USA

P. Dibbets-Schneider $\cdot$ B. van der Hiel $\cdot$ I. Al Younis

Department of Nuclear Medicine,

Leiden University Medical Center,

Leiden, The Netherlands repeated after 6 months of CRT. CRT response was defined as a decrease of $\geq 15 \%$ in LVESV.

Results Enrolled in the study were 90 patients $(72 \%$ men, $67 \pm 10$ years) with advanced heart failure. In 52 patients $(58 \%)$, the LV lead was positioned at the site of latest mechanical activation (concordant), and in 38 patients (42\%) the LV lead was positioned outside the site of latest mechanical activation (discordant). CRT response was significantly more often documented in patients with a concordant LV lead position than in patients with a discordant LV lead position ( $79 \%$ vs. $26 \%, p<0.01)$. After 6 months, patients with a concordant LV lead position showed significant improvement in LVEF, LVESV and LVEDV $(p<0.05)$, whereas patients with a discordant LV lead position showed no significant improvement in these variables.

Conclusion Patients with a concordant LV lead position showed significant improvement in LV volumes and LV systolic function, whereas patients with a discordant LV lead position showed no significant improvements.

Keywords Site of latest mechanical activation . LV lead . SPECT $\cdot$ Phase analysis $\cdot$ CRT

\section{Introduction}

Cardiac resynchronization therapy (CRT) is an established therapeutic option for patients with drug-refractory advanced heart failure and ventricular conduction delay $[1,2]$. The merits of CRT have been demonstrated in terms of morbidity and mortality in several randomized clinical trials which have currently included more than 4,000 patients diagnosed with moderate-to-severe heart failure [3-6]. Even though promising results have been reported, individual response to 
CRT varies with up to one-third of patients with heart failure showing no response to CRT [5, 7].

Different mechanisms play an important role in the response to CRT, such as preexisting mechanical dyssynchrony, location and extent of scarred myocardium and the position of the left ventricular (LV) pacing lead [7, 8]. The region of $\mathrm{LV}$ pacing and the area of latest mechanical activation, seem to be important factors in the prediction of outcome to CRT [9-14]. To date, the LV pacing lead is usually positioned in the lateral or posterolateral vein of the coronary sinus, since the largest haemodynamic response is observed when pacing the free lateral wall [9]. However, recent studies have also demonstrated that the region of latest mechanical activation may vary significantly in patients eligible for CRT [10, 14-16]. In previous studies using echocardiography patients with the LV lead positioned at the site of latest mechanical activation (concordant LV lead) have shown a superior response to CRT when compared to patients with the LV lead positioned outside the area of latest mechanical activation (discordant LV lead) [10, 14-16].

Phase analysis on gated myocardial perfusion single photon emission computed tomography (SPECT) (GMPS) has been developed to evaluate the presence and extent of mechanical dyssynchrony using an automatic and standardized approach [17, 18]. Recent developments have resulted in an integrated evaluation of mechanical dyssynchrony, the regional mechanical activation pattern and the site of latest mechanical activation using the same SPECT dataset. Hence, GMPS with phase analysis may provide important information for assessment of optimal LV lead position in patients referred for CRT. Currently, no study has been performed evaluating the use of GMPS to assess the preferred LV lead position. Moreover, the relationship between the site of latest mechanical activation as derived from GMPS, LV lead position and CRT response is unknown. Accordingly, in the current study we sought to evaluate the feasibility of GMPS for assessing the preferred LV lead position. In addition, we also aimed to evaluate the relationship between the site of latest mechanical activation, LV lead position and CRT response in patients with CRT.

\section{Materials and methods}

\section{Patient population and protocol}

Patients with advanced drug-refractory heart failure (New York Heart Association, NYHA, functional class III-IV), reduced $\mathrm{LV}$ systolic function (LV ejection fraction, LVEF, $\leq 35 \%$ ), and prolonged QRS interval ( $\geq 120 \mathrm{~ms}$ ) and sinus rhythm were consecutively included for implantation of a CRT device [1, 2]. Patients with decompensated heart failure, recent myocardial infarction (within 3 months of CRT device implantation) or who died during 6 months of follow-up were excluded. Ischaemic cardiomyopathy was defined as the presence of $\geq 50 \%$ stenosis in one or more of the major epicardial coronary arteries and/or previous myocardial infarction or percutaneous coronary intervention.

Prior to CRT device implantation, resting GMPS with ${ }^{99 \mathrm{~m}} \mathrm{Tc}$-tetrofosmin and transthoracic 2-D echocardiography were performed in all patients. Clinical status was evaluated by assessment of NYHA functional class. Resting myocardial perfusion imaging was performed to assess the presence and extent of myocardial infarction, and phase analyses of GMPS studies was used to assess the site of latest mechanical activation. Resting transthoracic 2-D echocardiography was performed to measure LVEF and LV volumes. Additionally, speckle tracking radial strain analysis on 2-D echocardiography was performed in a subset of 50 patients to validate the assessment of the site of latest mechanical activation as derived from GMPS studies.

After 6 months of CRT, assessment of clinical status and resting 2-D transthoracic echocardiography was repeated. Patients with a decrease of $\geq 15 \%$ in LV end-systolic volume (LVESV) were classified as responders to CRT, whereas patients without a decrease in LVESV $(<15 \%)$ were classified as nonresponders to CRT [19]. Consecutively, the relationship between the site of latest mechanical activation on GMPS, LV lead position and CRT response was evaluated.

\section{Gated myocardial perfusion SPECT}

Acquisition GMPS imaging with ${ }^{99} \mathrm{~m}$ Tc-tetrofosmin (500 MBq, MYOVIEW; General Electric Healthcare, UK) was performed at rest using a triple-head SPECT camera system (GCA 9300/HG; Toshiba Corporation, Tokyo, Japan) equipped with low-energy high-resolution collimators. A $20 \%$ window was used around the $140-\mathrm{keV}$ energy peak of ${ }^{99 \mathrm{~m}}$ Tc-tetrofosmin and 90 projections (step and shoot method, $35 \mathrm{~s} /$ projection, $64 \times 64$ matrix, total imaging time $23 \mathrm{~min}$ ) were obtained over a $360^{\circ}$ circular orbit. Acquisition involved 16 frames per cardiac cycle. Data were reconstructed by filtered back projection and reoriented into long- and short-axis projections perpendicular to the heart axis [20]. The short-axis slices were displayed in polar map format and they were normalized to maximal myocardial activity (100\%). No attenuation correction was used in this study. Two experienced observers who were blinded to other study data evaluated the SPECT studies. Cardiac segments with $<50 \%$ tracer uptake were considered as segments with a perfusion defect. The extent of myocardial perfusion defects $(<50 \%$ tracer uptake) was expressed as the percentage of the myocardium. Consecutively, reoriented gated short-axis images were submitted to the Emory Cardiac Toolbox (Emory University, Atlanta, 
GA) for phase analysis processing. Phase analysis measurements were performed at Emory University (blinded to echocardiographic and clinical data).

Phase analysis Phase analysis of GMPS studies was used to obtain the site of latest mechanical activation. Phase analysis of GMPS studies is based on the partial-volume effect wherein alterations in regional maximum counts are relative to myocardial wall thickening [21, 22]. A 3-D sampling algorithm is used to determine regional maximal counts per cardiac frame and is used to generate countbased wall thickening curves by approximation of first Fourier harmonics function. The phase angle represents the onset of mechanical activation per segment. The distribution of phase angles within the LV can be displayed in polar map or histogram format and provides quantitative parameters of global LV dyssynchrony, histogram bandwidth (includes $95 \%$ of the phase angles) and phase standard deviation (SD, standard deviation of phase distribution) $[17,18]$. In addition, phase analysis provides information on regional mechanical activation. For the current study, the area of latest mechanical activation was determined on GMPS studies using the six-segment model (septal, anteroseptal, anterior, lateral, posterior and inferior) [23]. Regions of interests (ROI) corresponding to the six-segment model were automatically placed on the phase polar map as shown in Fig. 1. Each ROI covered $45^{\circ}$ and six short-axis slices starting from the middle slice toward the base. As the 3 -D sampling algorithm collected one sample for every $9^{\circ}$, each ROI contained $5 \times 6=30$ samples. The mean phases of the six segments were calculated by averaging the phases of their 30 samples and then compared. The latest mechanically activated segment had the largest phase angles.

The intra- and interobserver reproducibility of phase analysis for assessment of the site of latest mechanical activation was determined in a subset of 30 patients, randomly selected from the patient population.
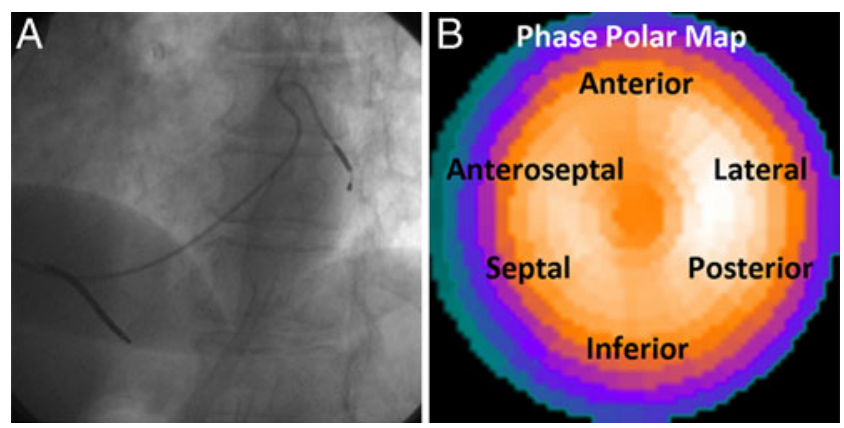

Fig. 1 The site of latest mechanical activation was assessed on GMPS studies (b) and related to the LV lead position on fluoroscopy (a). a LV lead positioned in the lateral cardiac region. $\mathbf{b}$ The region of latest mechanical activation was automatically calculated using phase analysis of GMPS studies. The region of latest mechanical activation was located on the phase polar map using the six-segment model [23]
Resting transthoracic echocardiography

Echocardiographic images were obtained with a commercially available system (Vivid Seven, General ElectricVingmed, Milwaukee, WI) in patients in the left lateral decubitus position. Data were acquired with a $3.5-\mathrm{MHz}$ transducer at a depth of $16 \mathrm{~cm}$ in the parasternal and apical views (standard long- and short-axis, two- and fourchamber images). Conventional 2-D images were obtained during breath-hold and saved in cine-loop format from three consecutive beats for off-line analysis (Echopac version 7.0.0; General Electric-Vingmed, Milwaukee, WI). From apical two- and four-chamber views, LVESV and LV end-diastolic volume (LVEDV) along with LVEF were measured using the biplane Simpson's approach [24]. Mitral regurgitation was assessed semiquantitatively from colour-flow Doppler images using the apical four-chamber views. Severity was scored according to the following scale: 1 mild jet area/left atrial area $<20 \%$ ), 2 moderate (jet area/left atrial area $20-45 \%$ ) and 3 severe (jet area/left atrial area $>45 \%)$ [25].

In 50 patients, the site of latest mechanical activation was assessed using 2-D speckle tracking radial strain analysis on baseline midventricular short-axis images. Images were recorded at a frame rate of at least 30 frames per second and time-frame curves were generated for six cardiac segments (Echopac version 7.0.0; General ElectricVingmed, Milwaukee, WI) in a similar manner to the GMPS studies. Finally, the time between QRS onset and peak radial strain of the cardiac segments was used to assess the site of latest mechanical activation [23].

\section{CRT implantation}

All leads were placed via the subclavian route and the right atrial and ventricular leads were placed conventionally [26]. With the use of a balloon catheter, a sinus venogram was obtained after occlusion of the coronary sinus. Subsequently, the LV pacing lead was inserted with an $8 \mathrm{~F}$ guiding catheter into the coronary sinus, preferably in the lateral or posterolateral vein. The $\mathrm{LV}$ pacing lead was positioned by an electrophysiologist who was blinded to other data. The V-V interval was not adjusted during the first 6 months of CRT.

LV lead position and the site of latest activation

LV lead positions were determined by an independent observer who was blinded to other data. The LV lead position was assessed on biplane fluoroscopy (which was obtained during the implantation procedure) using the left anterior oblique $\left(60^{\circ}\right)$ and right anterior oblique $\left(30^{\circ}\right)$ views. For this analysis, LV pacing leads that were positioned in the basal or mid-region of the LV were included and LV leads positioned 
at the cardiac apex were excluded from further analysis. Using the six-segment model [23], the LV lead positions were scored as anterior, lateral, posterior or inferior. Subsequently, the LV lead positions were related to the area of latest activation (six-segment model) as assessed by phase analysis of GMPS studies.

The LV lead position was considered concordant if the lead was positioned at the area of latest activation, and was considered discordant if the lead was positioned outside the area of latest activation.

Intra- and interobserver reproducibility for assessment of LV lead position was evaluated in a randomly selected subset of 30 patients. To assess intraobserver reproducibility, the position of the LV lead on biplane fluoroscopy was assessed twice by the same observer. To assess interobserver reproducibility, a second blinded observer assessed the LV lead position on biplane fluoroscopy.

\section{Statistical analysis}

Continuous data are presented as means \pm standard deviation and categorical data are presented as numbers and percentages. Differences in baseline characteristics between patients with concordant and those with discordant LV lead positions were evaluated with the unpaired Student's $t$ test (continuous data) and the chi-squared or Fisher's exact tests (categorical data). During follow-up, changes in continuous data were evaluated using the paired Student's $t$ test for both study groups. Agreement between GMPS with phase analysis and 2-D speckle tracking radial strain analysis for assessment of the site of latest mechanical activation was evaluated using Cohen's kappa statistics, and $k$ values were categorized as poor $(<0.40)$, moderate $(0.40-0.75)$ or good $(>0.75)$ agreement. In addition, Cohen's kappa statistics were used to evaluate intra- and interobserver reproducibility for the assessment of the site of latest mechanical activation using phase analysis in a subset of 30 randomly selected patients. Similarly, Cohen's kappa statistics were used to evaluate the intra- and interobserver reproducibility for assessment of the LV lead position on biplane fluoroscopy in 30 randomly selected patients. All tests were two-sided and for all analyses a $p$ values of $<0.05$ were considered statistically significant. Statistical analyses were performed with the SPSS software package, version 16.0 (SPSS, Chicago, IL).

\section{Results}

Patient population

A total of 95 consecutive patients were obtained from our ongoing clinical heart failure registry, of whom 50 had participated in previous work. Five patients were excluded because of (1) apical LV lead position $(n=3)$ or (2) cardiac death during 6 months of follow-up $(n=2)$. The baseline characteristics of the 90 heart failure patients $(72 \%$ men, mean age $67 \pm 10$ years) are listed in Table 1 . The mean NYHA functional class was $3.0 \pm 0.4$. Of the 90 patients, 62 (69\%) were diagnosed with ischaemic cardiomyopathy, and 28 (31\%) had nonischaemic cardiomyopathy. Patients showed reduced LV systolic function, with a mean LVEF of $26 \pm 8 \%$. The extent of the myocardial perfusion defect was $26 \pm 16 \%$ on average. Medication consisted of diuretics ( $90 \%$ of patients), angiotensin-converting enzyme inhibitors or angiotensin II antagonists ( $88 \%$ of patients) or betablockers (69\% of patients).

GMPS with phase analysis and LV lead position

The mean values of histogram bandwidth and phase SD were $139 \pm 77^{\circ}$ and $41 \pm 21^{\circ}$. The region of latest mechanical activation as assessed with GMPS was located in the posterior $(42.2 \%)$, lateral $(23.3 \%)$, inferior $(13.3 \%)$, anterior $(15.6 \%)$, anteroseptal (3\%) and septal $(2.3 \%)$ regions. Furthermore, good agreement was found between GMPS and 2-D speckle tracking radial strain analysis for assessment of the site of latest mechanical activation $(k=0.79$, total agreement of

Table 1 Baseline characteristics of the patient population $(n=90)$. Data are presented as means \pm standard deviation or as numbers (\%)

\begin{tabular}{ll}
\hline Characteristic & Value \\
\hline Age (years) & $67 \pm 10$ \\
Male gender & $65(72 \%)$ \\
Ischaemic heart failure & $62(69 \%)$ \\
NYHA functional class & $3.0 \pm 0.4$ \\
QRS duration (ms) & $161 \pm 36$ \\
Echocardiographic parameters & \\
LVEDV (ml) & $214 \pm 64$ \\
LVESV (ml) & $160 \pm 57$ \\
LVEF (\%) & $26 \pm 8$ \\
Scintigraphic parameters & \\
Perfusion defect in LV pacing region & $17(19 \%)$ \\
Extent of perfusion defect $(\%)$ & $26 \pm 16$ \\
Histogram bandwidth $\left({ }^{\circ}\right)$ & $139 \pm 77$ \\
Phase SD $\left(^{\circ}\right)$ & $41 \pm 21$ \\
Medication & \\
Diuretics & $81(90 \%)$ \\
Angiotensin-converting enzyme & $79(88 \%)$ \\
inhibitors/angiotensin II antagonists & $62(69 \%)$ \\
Beta-blockers & $40(44 \%)$ \\
Spironolactone & $55(61 \%)$ \\
Statins &
\end{tabular}


$86 \%)$. Good intraobserver $(k=0.96$, total agreement of $93 \%)$ and interobserver $(k=0.92$, total agreement of $87 \%)$ reproducibility of the phase analysis was observed for assessment of the site of latest mechanical activation. CRT device and LV lead implantation were successful in all patients without major complications. The LV pacing lead was positioned in the lateral ( $44.4 \%$ of patients), posterior (50.0\% of patients) or anterior (5.6\% of patients) regions. Good intraobserver $(k=0.82$, total agreement of $90 \%)$ and interobserver $(k=0.76$, total agreement of $87 \%)$ reproducibility for assessment of LV lead position on fluoroscopy was observed. LV lead position was concordant in 52 patients (58\%) and discordant in $38(42 \%)$, as shown in Table 2. No significant differences were observed in demographic, clinical or echocardiographic variables between patients with concordant or discordant LV lead position. In addition, no differences were found for histogram bandwidth and phase SD between the two groups. Patients with concordant and discordant LV lead positions showed no significant differences in perfusion defects located in the LV pacing region (13\% vs. $26 \%, p=$ NS). Additionally, no differences were found between patients with concordant and discordant LV lead positions in the extent of myocardial perfusion defects $(22.9 \pm 14.1 \%$ vs. $29.3 \pm 18.1 \%, p=\mathrm{NS})$. The extent of myocardial perfusion defects was significantly smaller in patients with a CRT response than in those without a CRT response

Table 2 Baseline characteristics of patients with concordant and discordant LV lead positions. Data are presented as means \pm standard deviation or as numbers (\%)

\begin{tabular}{llll}
\hline $\begin{array}{l}\text { Baseline } \\
\text { characteristics }\end{array}$ & $\begin{array}{l}\text { Concordant LV } \\
\text { lead position } \\
(n=52)\end{array}$ & $\begin{array}{l}\text { Discordant LV } \\
\text { lead position } \\
(n=38)\end{array}$ & $\begin{array}{l}p \\
\text { value }\end{array}$ \\
\hline Age (years) & $68 \pm 10$ & $66 \pm 11$ & 0.5 \\
Male gender & $37(71 \%)$ & $28(74 \%)$ & 0.8 \\
Ischaemic heart failure & $38(73 \%)$ & $24(63 \%)$ & 0.4 \\
NYHA functional class & $3.0 \pm 0.4$ & $3.0 \pm 0.4$ & 1.0 \\
QRS duration (ms) & $168 \pm 35$ & $153 \pm 33$ & 0.1 \\
LVEDV (ml) & $214 \pm 67$ & $213 \pm 62$ & 1.0 \\
LVESV (ml) & $160 \pm 57$ & $161 \pm 57$ & 0.9 \\
LVEF (\%) & $27 \pm 8$ & $26 \pm 8$ & 0.7 \\
Mitral regurgitation & $19(37 \%)$ & $19(50 \%)$ & 0.2 \\
$\quad$ (moderate/severe) & $126 \pm 67$ & $157 \pm 86$ & 0.1 \\
$\begin{array}{l}\text { Histogram bandwidth }\left({ }^{\circ}\right) \\
\left.\text { Phase SD ( }{ }^{\circ}\right)\end{array}$ & $38 \pm 20$ & $45 \pm 21$ & 0.1 \\
$\begin{array}{l}\text { Perfusion defect LV } \\
\text { pacing region }\end{array}$ & $7(13 \%)$ & $10(26 \%)$ & 0.2 \\
Extent of perfusion & $23 \pm 14$ & $29 \pm 18$ & 0.1 \\
$\quad$ defect $(\%)$ & & $10(26 \%)$ & $<0.01$ \\
CRT response after & $41(79 \%)$ & & \\
$\quad 6$ months & & & \\
\hline
\end{tabular}

$(21.1 \pm 12.3 \%$ vs. $31.6 \pm 18.7 \%, p<0.05)$. Furthermore, the percentage of CRT responders was significantly higher among patients with a concordant LV lead position than among those with a discordant LV lead position (79\% vs. $26 \%, p<0.01)$. Patient examples with a concordant and a discordant LV lead position are shown in Fig. 2. Of note, 11 patients with a concordant LV lead position showed no response to CRT after 6 months. Of these patients, seven showed severe perfusion defects at the region of LV pacing.

Baseline and 6 months follow-up

The total patient population showed a significant improvement in NYHA functional class from $3.0 \pm 0.4$ to $2.5 \pm 0.7$ $(p<0.05)$. In addition, patients showed a significant improvement in echocardiographic variables, including LVESV $(160 \pm 57 \mathrm{ml}$ vs. $137 \pm 55 \mathrm{ml}, p<0.05)$, LVEDV $(214 \pm 64 \mathrm{ml}$ vs. $197 \pm 64 \mathrm{ml}, p<0.05)$ and LVEF $(26 \pm 8 \%$ vs. $32 \pm 11 \%, p<0.05$ ). After 6 months of CRT, patients with a concordant LV lead position showed significant improvement in LVESV $(159 \pm 57 \mathrm{ml}$ vs. $125 \pm 54 \mathrm{ml}, p<$ $0.05)$, LVEDV $(214 \pm 67 \mathrm{ml}$ vs. $188 \pm 62 \mathrm{ml}, p<0.05)$ and LVEF $(27 \pm 8 \%$ vs. $35 \pm 12 \%, p<0.05)$, as illustrated in Fig. 3. Additionally, an improvement in NYHA functional class was observed in patients with a concordant LV lead position ( $3.0 \pm 0.4$ vs. $2.3 \pm 0.7, p<0.05)$. However, patients with a discordant LV lead position showed no improvement in NYHA functional class $(3.0 \pm 0.4$ vs. $2.7 \pm 0.7, p=$ NS). In addition, LVESV ( $161 \pm 57 \mathrm{ml}$ vs. $153 \pm 53 \mathrm{ml}, p=$ NS), LVEDV (213 $\pm 62 \mathrm{ml}$ vs. $210 \pm 65 \mathrm{ml}, p=\mathrm{NS})$ and LVEF ( $26 \pm 8 \%$ vs. $28 \pm 9 \%, p=\mathrm{NS}$ ) showed no significant improvement after 6 months of CRT in patients with a discordant LV lead position, as shown in Fig. 3.

\section{Discussion}

The main findings of this study can be summarized as follows: patients with a concordant LV lead position showed significant LV reverse remodelling and improvement in LV systolic function, whereas patients with a discordant LV lead position showed no significant improvements. Accordingly, GMPS with phase analysis is a feasible technique to identify the preferred LV lead position in patients referred for CRT.

The rationale for CRT is based on improving the intrinsic electrical ventricular conductance by pacing the ventricles in a synchronized manner $[1,2]$. To optimize synchronicity of the LV contraction, the LV pacing lead should be targeted at the region of latest mechanical activation [9-13]. It is important to note that the region of latest mechanical activation may vary significantly in 

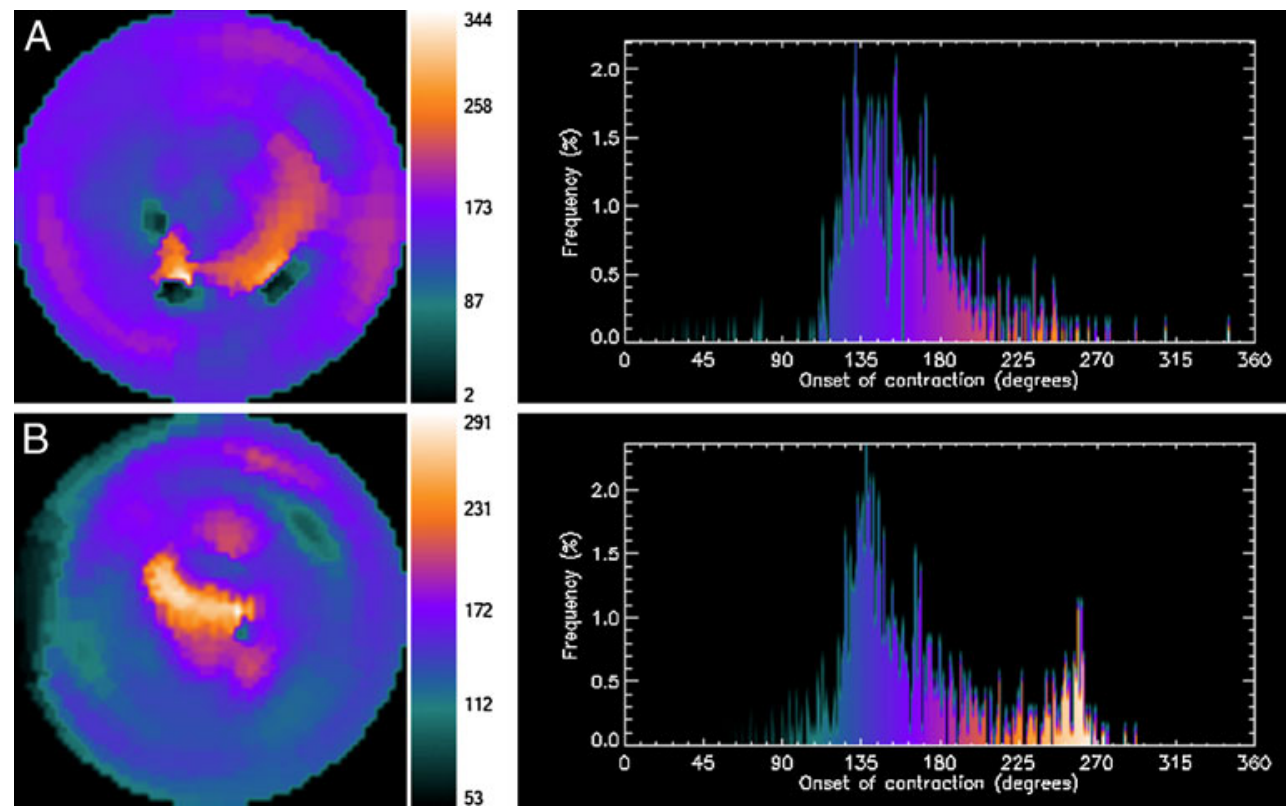

Fig. 2 Area of latest mechanical activation as assessed by phase analysis of GMPS studies. a LV lead positioned at the area of latest activation (concordant LV lead position). The area of latest activation Is located in the lateral segment. The patient showed a significant improvement in LVESV (139 ml vs. $86 \mathrm{ml}$ ) and LVEF (32\% vs. 44\%)

patients with heart failure, with the posterolateral region as the most common site of latest activation [10, 14-16]. The study by Becker et al. [16] determined the effect of LV lead position on reverse remodelling and LV function as assessed by echocardiography in 47 patients with heart failure and found that the site of latest mechanical activation differed significantly among the patients. The site of latest mechanical activation was predominantly located in the posterolateral region of the ventricle $(60 \%$ of patients). Likewise, the current study showed that the after 6 months of CRT. b LV lead positioned outside the area of latest activation (discordant LV lead position). The area of latest activation is located in the anterior segment, whereas the LV lead is positioned in the posterior segment. The patient showed no improvement in LVESV $(124 \mathrm{ml}$ vs. $153 \mathrm{ml})$ or LVEF $(27 \%$ vs. $22 \%)$ after 6 months of CRT

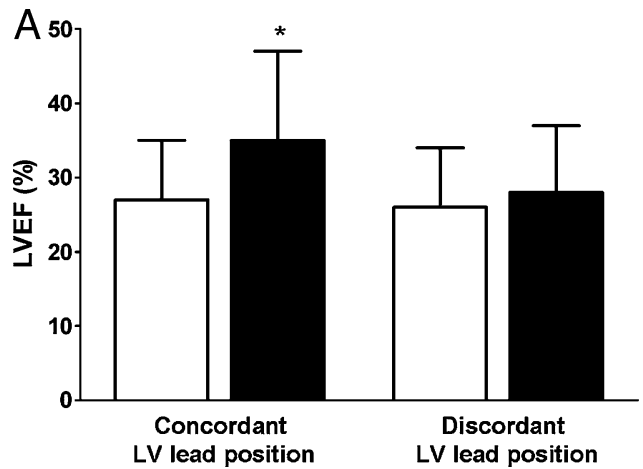

Fig. 3 Response to CRT in patients with concordant $(n=52)$ and discordant $(n=38)$ LV lead positions. Baseline (white bars) and 6month follow-up (black bars) echocardiographic measurements are shown. a Patients with a concordant LV lead position showed significant improvement in $\operatorname{LVEF}(27 \pm 8 \%$ vs. $35 \pm 12 \%, p<0.05)$, and patients with a discordant LV lead position showed no significant posterolateral cardiac segments were the most common site of latest mechanical activation ( $66 \%$ of patients).

Additionally, the relationship between LV lead position, the site of mechanical delay and CRT outcome has been evaluated in several studies [9-16]. An important study was performed by Becker et al. [16] who evaluated the efficacy of CRT in patients with optimal $(n=28)$ or suboptimal $(n=$ 19) LV lead position. The position of the LV lead was considered optimal if it was concordant with (one segment or less between the LV pacing region and the segment with

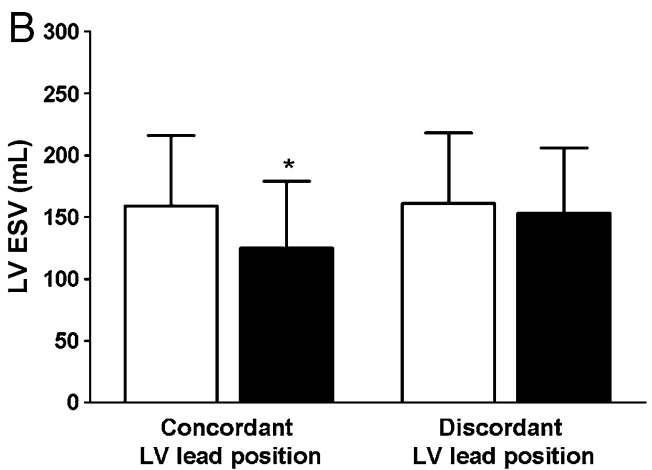

improvement in LVEF ( $26 \pm 8 \%$ vs. $28 \pm 9 \%, p=\mathrm{NS})$. b Patients with a concordant LV lead position showed significant improvement in LVESV $(159 \pm 57 \mathrm{ml}$ vs. $125 \pm 54 \mathrm{ml}, p<0.05)$, whereas patients with a discordant LV lead position showed no significant improvement in LVESV (161 $\pm 57 \mathrm{ml}$ vs. $153 \pm 53 \mathrm{ml}, p=\mathrm{NS})$ 
latest mechanical activation) the cardiac segment showing the latest mechanical contraction. Circumferential strain analysis on echocardiography was used to assess the site of delayed mechanical activation. After 10 months of followup, patients with an optimal LV lead position showed a significantly greater decrease in LVESV and LVEDV as well as a significant improvement in LVEF, as compared to patients with a suboptimal LV lead position (all, $p<0.01$ ).

Recently, Ypenburg et al. [14] evaluated 6-month echocardiographic response to CRT as well as long-term outcome in patients with concordant $(n=153)$ and discordant LV lead $(n=91)$ position. Speckle tracking radial strain analysis on 2-D echocardiography was used to determine the region of latest mechanical delay. After 6 months of CRT, patients with a concordant LV lead position showed a significant decrease in LV volumes as well as a significant improvement in LVEF as compared to patients with a discordant LV lead position (all, $p<0.01$ ). Importantly, a concordant LV lead position was an independent predictor of combined endpoint of hospitalization and all-cause mortality. Similarly, the current study demonstrated that patients with a concordant LV lead position showed greater improvement in LVEF after 6 months of CRT $(27 \pm 8 \%$ vs. $35 \pm 12 \%, p<0.05)$ than patients with a discordant LV lead position ( $26 \pm 8 \%$ vs. $28 \pm 9 \%, p=\mathrm{NS})$. Additionally, considerable reverse remodelling, as reflected by a significant decrease in LVESV and LVEDV, was observed in patients with the LV lead positioned at the site of latest activation as derived from GMPS with phase analysis. The findings demonstrate that CRT response is related to LV lead position and the region of latest mechanical contraction. More specifically, these observations support the hypothesis that resynchronization of the LV, induced by pacing at the region of latest mechanical activation, exerts positive effects on ventricular geometry and function.

Of interest, a small number of patients with the LV lead placed outside the region of latest activation responded positively to CRT. In these patients, the distance between the region of latest activation and the LV lead position (as reflected by the number of cardiac segments in between) was minimal (up to two cardiac segments). As a consequence, the contraction pattern may have become less dyssynchronous in these patients. Additionally, 11 patients (21\%) did not show a response to CRT despite the fact that the LV lead was positioned at the latest activated area. This observation may be explained by the fact that extensive perfusion defects were located at or near the site of latest mechanical activation in this subset of patients. It has been demonstrated that the extent and location of scarred myocardium play an important role in response to CRT [26-28]. Bleeker et al. [26] performed an important study evaluating the effect of posterolateral scar tissue on response to CRT in 40 consecutive patients with advanced heart failure. The study showed that patients with posterolateral scar tissue on MRI showed a significantly lower response rate than patients without posterolateral scar tissue $(14 \%$ vs. $81 \%, p<0.05)$. Similarly, the current study demonstrated that severe perfusion defects at or adjacent to the region of latest mechanical activation were associated with lack of response to CRT. Patients with a concordant LV lead position but with severe perfusion defects at the region of $\mathrm{LV}$ pacing showed no response to CRT. Furthermore, the study showed that the extent of myocardial perfusion defects was significantly smaller in patients with response to CRT than in patients with no response to CRT. Accordingly, location and extent of scarred myocardium play an important role in the likelihood of response to CRT.

At present, a lack of response to CRT has been associated with an absence of preexisting mechanical dyssynchrony, location and extent of scarred myocardium (particularly at the LV pacing region) and a suboptimal LV lead position [7, 8, 26-28]. For this reason, phase analysis of GMPS studies has attracted increasing interest for the evaluation of CRT patients as it allows an integrated evaluation of mechanical dyssynchrony, regional activation pattern and myocardial infarction [17, 18]. More specifically, the presence of infarcted myocardium at or adjacent to the region of maximal mechanical delay can be evaluated using SPECT datasets. Accordingly, phase analysis of GMPS studies is a feasible technique to identify the preferred LV lead position.

At present, different noninvasive imaging techniques are available for the evaluation of patients referred for CRT, including echocardiography, MRI as well as nuclear imaging. A comprehensive evaluation of patients with CRT can be performed by each of these imaging techniques as they provide information on preexisting LV mechanical dyssynchrony, the presence and location of myocardial infarction as well as the site of latest mechanical activation. As they all provide useful information, the choice of one of these techniques is eventually determined by local expertise and availability.

Some study limitations need to be acknowledged. First, the study findings were based on a relatively small number of patients who were referred for CRT. The current study, however, was only a feasibility study evaluating the role of phase analysis of GMPS studies in the assessment of optimal LV lead position in patients with advanced heart failure. The novelty of the present study relates to the evaluation of response to CRT in patients with concordant and discordant LV lead position as assessed by phase analysis of GMPS studies. A second limitation is the fact that long-term effects of CRT on mortality and/or morbidity rates were not reported in the current study as the patient population was too small for long-term outcome analysis. 


\section{Conclusion}

Patients with a concordant LV lead position showed significant improvement in LV volumes and LV systolic function, whereas patients with a discordant LV lead position showed no significant improvement in LV volumes or LV systolic function. Accordingly, phase analysis of GMPS studies is a feasible technique that can be used to identify the preferred LV lead position in patients referred for CRT.

Acknowledgments Mark J. Boogers is supported by the Dutch Heart Foundation (grant number 2006T102). This study was also supported in part by a research project funded by the NIH (1R01HL094438-01A1, PI: Ji Chen).

Conflicts of interest Martin J. Schalij received research grants from Boston Scientific, Medtronic and Biotronik. Jeroen J. Bax received research grants from Medtronic, Boston Scientific, Biotronik, Edwards Lifesciences, BMS Medical Imaging, St. Jude Medical and GE Healthcare. Ji Chen and Ernest V. Garcia receive royalties from the sale of the Emory Cardiac Toolbox. The terms of this arrangement have been reviewed and approved by Emory University in accordance with its conflict-of-interest practice.

Open Access This article is distributed under the terms of the Creative Commons Attribution Noncommercial License which permits any noncommercial use, distribution, and reproduction in any medium, provided the original author(s) and source are credited.

\section{References}

1. Hunt SA, Abraham WT, Chin MH, Feldman AM, Francis GS, Ganiats TG, et al. 2009 focused update incorporated into the ACC/AHA 2005 guidelines for the diagnosis and management of heart failure in adults: a report of the American College of Cardiology Foundation/American Heart Association Task Force on practice guidelines: developed in collaboration with the International Society for Heart and Lung Transplantation. Circulation 2009;119(14):e391-479.

2. Epstein AE, DiMarco JP, Ellenbogen KA, Estes NA III, Freedman RA, Gettes LS, et al. ACC/AHA/HRS 2008 Guidelines for devicebased therapy of cardiac rhythm abnormalities: a report of the American College of Cardiology/American Heart Association Task Force on Practice Guidelines (Writing Committee to Revise the ACC/ AHA/NASPE 2002 guideline update for implantation of cardiac pacemakers and antiarrhythmia devices): developed in collaboration with the American Association for Thoracic Surgery and Society of Thoracic Surgeons. Circulation. 2008;117(21):e350-408.

3. Cazeau S, Leclercq C, Lavergne T, Walker S, Varma C, Linde C, et al. Effects of multisite biventricular pacing in patients with heart failure and intraventricular conduction delay. N Engl J Med. 2001;344(12):873-80.

4. Abraham WT, Fisher WG, Smith AL, Delurgio DB, Leon AR, Loh $\mathrm{E}$, et al. Cardiac resynchronization in chronic heart failure. $\mathrm{N}$ Engl J Med. 2002;346(24):1845-53.

5. Bristow MR, Saxon LA, Boehmer J, Krueger S, Kass DA, De MT, et al. Cardiac-resynchronization therapy with or without an implantable defibrillator in advanced chronic heart failure. N Engl J Med. 2004;350(21):2140-50.
6. Cleland JG, Daubert JC, Erdmann E, Freemantle N, Gras D, Kappenberger L, et al. The effect of cardiac resynchronization on morbidity and mortality in heart failure. N Engl J Med. 2005;352 (15):1539-49.

7. Bax JJ, Abraham T, Barold SS, Breithardt OA, Fung JW, Garrigue $\mathrm{S}$, et al. Cardiac resynchronization therapy: Part 1-issues before device implantation. J Am Coll Cardiol. 2005;46(12):2153-67.

8. Bax JJ, Abraham T, Barold SS, Breithardt OA, Fung JW, Garrigue $\mathrm{S}$, et al. Cardiac resynchronization therapy: part 2-issues during and after device implantation and unresolved questions. J Am Coll Cardiol. 2005;46(12):2168-82.

9. Butter C, Auricchio A, Stellbrink C, Fleck E, Ding J, Yu Y, et al. Effect of resynchronization therapy stimulation site on the systolic function of heart failure patients. Circulation. 2001;104(25):3026-9.

10. Ansalone G, Giannantoni P, Ricci R, Trambaiolo P, Fedele F, Santini M. Doppler myocardial imaging to evaluate the effectiveness of pacing sites in patients receiving biventricular pacing. $\mathrm{J}$ Am Coll Cardiol. 2002;39(3):489-99.

11. Gasparini M, Mantica M, Galimberti P, Bocciolone M, Genovese $\mathrm{L}$, Mangiavacchi $\mathrm{M}$, et al. Is the left ventricular lateral wall the best lead implantation site for cardiac resynchronization therapy? Pacing Clin Electrophysiol. 2003;26(1 Pt 2):162-8.

12. Dekker AL, Phelps B, Dijkman B, van der Nagel T, van Veen FH, Geskes GG, et al. Epicardial left ventricular lead placement for cardiac resynchronization therapy: optimal pace site selection with pressure-volume loops. J Thorac Cardiovasc Surg. 2004;127 (6): $1641-7$

13. Lane RE, Chow AW, Mayet J, Francis DP, Peters NS, Schilling $\mathrm{RJ}$, et al. The interaction of interventricular pacing intervals and left ventricular lead position during temporary biventricular pacing evaluated by tissue Doppler imaging. Heart. 2007;93 (11):1426-32.

14. Ypenburg C, Van Bommel RJ, Delgado V, Mollema SA, Bleeker $\mathrm{GB}$, Boersma E, et al. Optimal left ventricular lead position predicts reverse remodeling and survival after cardiac resynchronization therapy. J Am Coll Cardiol. 2008;52(17):1402-9.

15. Murphy RT, Sigurdsson G, Mulamalla S, Agler D, Popovic ZB, Starling RC, et al. Tissue synchronization imaging and optimal left ventricular pacing site in cardiac resynchronization therapy. Am J Cardiol. 2006;97(11):1615-21.

16. Becker M, Kramann R, Franke A, Breithardt OA, Heussen N, Knackstedt $C$, et al. Impact of left ventricular lead position in cardiac resynchronization therapy on left ventricular remodelling. A circumferential strain analysis based on 2D echocardiography. Eur Heart J. 2007;28(10):1211-20.

17. Chen J, Garcia EV, Folks RD, Cooke CD, Faber TL, Tauxe EL, et al. Onset of left ventricular mechanical contraction as determined by phase analysis of ECG-gated myocardial perfusion SPECT imaging: development of a diagnostic tool for assessment of cardiac mechanical dyssynchrony. J Nucl Cardiol. 2005;12 (6):687-95.

18. Chen J, Henneman MM, Trimble MA, Bax JJ, Borges-Neto S, Iskandrian $\mathrm{AE}$, et al. Assessment of left ventricular mechanical dyssynchrony by phase analysis of ECG-gated SPECT myocardial perfusion imaging. J Nucl Cardiol. 2008;15(1):127-36.

19. Bax JJ, Bleeker GB, Marwick TH, Molhoek SG, Boersma E, Steendijk P, et al. Left ventricular dyssynchrony predicts response and prognosis after cardiac resynchronization therapy. J Am Coll Cardiol. 2004;44(9):1834-40.

20. Cerqueira MD, Weissman NJ, Dilsizian V, Jacobs AK, Kaul S, Laskey WK, et al. Standardized myocardial segmentation and nomenclature for tomographic imaging of the heart: a statement for healthcare professionals from the Cardiac Imaging Committee of the Council on Clinical Cardiology of the American Heart Association. Circulation. 2002;105(4):539-42. 
21. Hoffman EJ, Huang SC, Phelps ME. Quantitation in positron emission computed tomography: 1. Effect of object size. J Comput Assist Tomogr. 1979;3(3):299-308.

22. Galt JR, Garcia EV, Robbins WL. Effects of myocardial wall thickness on SPECT quantification. IEEE Trans Med Imaging. 1990;9(2):144-50.

23. Suffoletto MS, Dohi K, Cannesson M, Saba S, Gorcsan J III. Novel speckle-tracking radial strain from routine black-and-white echocardiographic images to quantify dyssynchrony and predict response to cardiac resynchronization therapy. Circulation. 2006;113(7):960-8.

24. Schiller NB, Shah PM, Crawford M, DeMaria A, Devereux R, Feigenbaum $\mathrm{H}$, et al. Recommendations for quantitation of the left ventricle by two-dimensional echocardiography. American Society of Echocardiography Committee on Standards, Subcommittee on Quantitation of Two-Dimensional Echocardiograms. J Am Soc Echocardiogr. 1989;2(5):358-67.

25. Bonow RO, Carabello BA, Chatterjee K, de Leon AC Jr, Faxon DP, Freed MD, et al. 2008 Focused update incorporated into the ACC/AHA 2006 guidelines for the management of patients with valvular heart disease: a report of the American College of Cardiology/American Heart Association Task Force on Practice Guidelines (Writing Committee to Revise the 1998 Guidelines for the Management of Patients With Valvular Heart Disease): endorsed by the Society of Cardiovascular Anesthesiologists, Society for Cardiovascular Angiography and Interventions, and Society of Thoracic Surgeons. Circulation. 2008;118(15):e523661.

26. Bleeker GB, Kaandorp TA, Lamb HJ, Boersma E, Steendijk P, de Roos A. Effect of posterolateral scar tissue on clinical and echocardiographic improvement after cardiac resynchronization therapy. Circulation. 2006;113(7):969-76.

27. Ypenburg C, Schalij MJ, Bleeker GB, Steendijk P, Boersma E, Dibbets-Schneider P, et al. Extent of viability to predict response to cardiac resynchronization therapy in ischemic heart failure patients. J Nucl Med. 2006;47(10):1565-70.

28. Ypenburg C, Schalij MJ, Bleeker GB, Steendijk P, Boersma E, Dibbets-Schneider P, et al. Impact of viability and scar tissue on response to cardiac resynchronization therapy in ischaemic heart failure patients. Eur Heart J. 2007;28(1):33-41. 